\title{
Voltage-Gated Potassium Channel Antibody Paraneoplastic Limbic Encephalitis Associated with Acute Myeloid Leukemia
}

\author{
Marion Alcantara ${ }^{a} \quad$ Omar Bennani $^{\mathrm{b}} \quad$ Pierre Verdure $^{\mathrm{b}}$ \\ Stéphane Leprêtre ${ }^{a}$ Hervé Tilly $^{a}$ Fabrice Jardin ${ }^{a}$ \\ ${ }^{a}$ Department of Hematology, Centre Henri Becquerel, and ${ }^{b}$ Department of Neurology, \\ CHU Charles Nicolle, Rouen, France
}

\section{Key Words}

Limbic encephalitis · Paraneoplastic syndrome $\cdot$ Acute myeloid leukemia

\begin{abstract}
Among paraneoplastic syndromes (PNS) associated with malignant hemopathies, there are few reports of PNS of the central nervous system and most of them are associated with lymphomas. Limbic encephalitis is a rare neurological syndrome classically diagnosed in the context of PNS. We report the case of a 81-year-old man who presented with a relapsed acute myeloid leukemia (AML) with minimal maturation. He was admitted for confusion with unfavorable evolution as he presented a rapidly progressive dementia resulting in death. A brain magnetic resonance imaging, performed 2 months after the onset, was considered normal. An electroencephalogram showed non-specific bilateral slow waves. We received the results of the blood screening of neuronal autoantibodies after the patient's death and detected the presence of anti-voltage-gated potassium channel (VGKC) antibodies at 102 $\mathrm{pmol} / \mathrm{l}$ (normal at $<30 \mathrm{pmol} / \mathrm{l}$ ). Other etiologic studies, including the screening for another cause of rapidly progressive dementia, were negative. To our knowledge, this is the first case of anti-VGKC paraneoplastic limbic encephalitis related to AML.
\end{abstract}

\section{Introduction}

Limbic encephalitis is a rare neurological disorder characterized by amnesia, seizures, and psychiatric disturbances, associated with anti-neuronal antibodies that can target either 
Alcantara et al.: Voltage-Gated Potassium Channel Antibody Paraneoplastic Limbic Encephalitis Associated with Acute Myeloid Leukemia

intracellular or neuronal cell surface antigens as voltage-gated potassium channel (VGKC) [1]. Limbic encephalitis is the second most common non-prion diagnostic of rapidly progressive dementia [2]. It is usually associated with an underlying neoplasm in the context of a paraneoplastic syndrome (PNS) [1].

Here, we describe the first case of anti-VGKC paraneoplastic limbic encephalitis in a patient presenting with a rapidly progressive dementia, in conjunction with a relapsed acute myeloid leukemia (AML).

\section{Case Report}

In December 2009, a 79-year-old man was diagnosed with an AML with minimal maturation (AML 1), normal karyotype, activating mutation of the FMS-like tyrosine kinase-3 internal tandem duplication (FLT3-ITD), and no mutation of NPM1, CEBP $\alpha 1$, or CEBP $\alpha 2$.

The patient had neither prior health problems nor cognitive disturbances. He received induction treatment with idarubicin and cytarabine, achieving complete response.

Induction was followed by consolidation treatment: six courses of daunorubicin and cytarabine, the last one performed in July 2010.

In March 2011, anemia, thrombocytopenia and hyperleukocytosis recurred, leading to the diagnosis of relapsed AML 1 with persistence of FLT3-ITD. The patient received an oral FLT3 inhibitor, achieving complete response. He was treated with $10 \mathrm{mg}$ of amlodipine and 16 IU of insulatard.

In June 2011, evolution occurred with onset of confusion. He lost orientation to place and time, and his nycthemeral rhythm was disturbed expressing agitation in the night and vigilance disturbances. Neurological examination found no motor/sensitive deficiency, the reflexes were not perceived but an extrapyramidal rigidity was noted. Basic blood laboratory tests revealed an isolated hyponatremia $(125 \mathrm{mmol} / \mathrm{l})$. No evident etiology was found to explain this disturbance. Laboratory examination of the cerebrospinal fluid revealed an elevated protein concentration $(0.69 \mathrm{~g} / \mathrm{l})$, normal cell count and no malignant cells, normal lactate, normal glucose, negative 14.3.3 protein, negative screening for herpes simplex virus and HHV6 by polymerase chain reaction, and sterile bacterial, viral or fungal cultures. Brain magnetic resonance imaging (MRI), 2 months after the onset, was considered normal. An electroencephalogram (EEG) showed non-specific bilateral slow waves. Serum evaluation for antithyroid antibodies was negative. Screening for plasmatic antinuclear antibodies and antineutrophil cytoplasmic antibodies was negative. There was no vitamin deficiency. Despite stopping all treatments and natremia correction, the patient presented with a rapidly progressive dementia with hallucinations, extrapyramidal and pyramidal rigidity affecting the limbs and the axis, without seizures or myoclonus, resulting in death in September 2011. The family refused an autopsy.

We received the results of the blood screening of neuronal autoantibodies after the patient's death and detected the presence of anti-VGKC antibodies at $102 \mathrm{pmol} / \mathrm{l}$ (normal at $<30 \mathrm{pmol} / \mathrm{l}$ ), on a radioimmunoassay. Anti-Lgi1 and anti-Caspr2 antibodies were both negative. All other tested neuronal antibodies remained negative. The diagnosis of postmortem anti-VGCK paraneoplastic limbic encephalitis was finally made. 
Alcantara et al.: Voltage-Gated Potassium Channel Antibody Paraneoplastic Limbic Encephalitis Associated with Acute Myeloid Leukemia

\section{Discussion}

This patient presented a PNS of the central nervous system according to the diagnostic criteria for PNS [3]: a non-classic syndrome with positive neuronal antibodies and cancer that develops within 5 years after the diagnosis of the neurological disorder.

Syndromes associated with antibodies against VGKC are typical limbic encephalitis and Morvan's syndrome, defined by the association of psychiatric symptoms, hallucinations, peripheral nerve hyperexcitability, hyperhydrosis, and other symptoms of autonomic dysfunction. In classical limbic encephalitis, patients presented with mood and sleep disturbances, seizures, hallucinations, and short-term memory loss that can progress to dementia, as in our case [1]. MRI was normal, as is the case in up to $45 \%$ of patients with limbic encephalitis with VGKC complex antibodies [4].

VGKC is a complex linked to neuronal cell surface. Most of the antibodies directed towards the VGKC complexes are binding to other proteins which are components of the complexes, mainly to Lgi1 and Caspr2, and less frequently, to contactin-2 [4]. As all the components of the VGKC complexes are not known, it seems important to resort to cellbased assays for Lgi1, Caspr2, and contactin-2 and the radioimmunoassay for VGKC complexes antibodies, when limbic encephalitis is suspected.

Only $30 \%$ of patients with antibodies against VGKC have tumors, and the cases reported in the literature are more often associated with thymoma, small cell lung cancer and other rare tumors [1]. We performed a chest X-ray to exclude the possibility that our patient harbored an occult cancer in addition to AML 1 but no suspicious lesion was detected. Other etiologic studies included the screening for another cause of rapidly progressive dementia [2] (for results, see text mentioned above). Prion disease was ruled out by a normal MRI and EEG 2 months after the onset, and was associated with negative controlled 14.3.3 protein. The iatrogenic hypothesis was no longer followed due to the lack of improvement after stopping all treatments. Moreover, pharmacovigilance research showed that no treatment received during the course of this disease was considered to be effective.

In this case, we could not treat the patient as the diagnosis was made postmortem. Treatment options include corticosteroids, intravenous immunoglobulin or plasma exchange; about $80 \%$ of patients respond to these treatments [1].

To our knowledge, this is the first case of anti-VGKC paraneoplastic limbic encephalitis associated with AML. Previously, there has been only one reported case of PNS of the central nervous system with VGKC antibodies (a Charcot-Marie-Tooth syndrome) related to leukemia [5]. Two other cases were reported with limbic encephalitis in patients with nonHodgkin lymphoma [6, 7]. Most findings of limbic encephalitis in malignant hemopathies are associated with Hodgkin lymphoma and none are reported to be caused by VGKC antibodies [8-10].

More frequent screening looking for PNS, including anti-neuronal and anti-VGKC antibodies, should be performed on patients with malignant hemopathies when faced with atypical syndromes of the central nervous system.

\section{References}

1 Dalmau J, Rosenfeld MR: Paraneoplastic syndromes of the CNS. Lancet Neurol 2008;7:327-340.

2 Geschwind MD, Shu H, Haman A, Sejvar JJ, Miller BL: Rapidly progressive dementia. Ann Neurol 2008;64:97-108.

-3 Graus F, Delattre JY, Antoine JC, et al: Recommended diagnostic criteria for paraneoplastic neurological syndromes. J Neurol Neurosurg Psychiatr 2004;75:1135-1140. 
4 Vincent A, Bien CG, Irani SR, Waters P: Autoantibodies associated with diseases of the CNS: new developments and future challenges. Lancet Neurol 2011;10:759-772.

-5 Blatt J, Greenwood R, Weig S, Rao K, Fedoriw GD, Dent G: Isolated central nervous system relapse in an adolescent with acute myelomonocytic leukemia, Charcot Marie Tooth syndrome, and paraneoplastic autoantibody. J Pediatr Hematol Oncol 2010;32:571-573.

$\checkmark 6$ Kanemitsu M, Ueno H, Naito K: A case of paraneoplastic limbic encephalitis associated with malignant lymphoma presenting with a late-onset bilateral thalamic lesion. Brain Nerve 2012;64:71-77.

-7 Kawashima S, Okita K, Yamawaki T, Matsukawa N, Ojika K: Case of paraneoplastic limbic encephalitis associated with malignant B cell lymphoma. Brain Nerve 2009;61:208-212.

-8 Zandi MS, Irani SR, Follows G, Moody AM, Molyneux P, Vincent A: Limbic encephalitis associated with antibodies to the NMDA receptor in Hodgkin lymphoma. Neurology 2009;73:2039-2040.

-9 Serratrice J, Amri AB, Bouabdallah R, Ceccaldi M, Weiller PJ: Relapsing meningitis and confusion: limbic encephalitis revealing Hodgkin's disease. Rev Neurol 2009;165:76-80.

10 Laffon M, Giordana C, Almairac F, Benchetrit M, Thomas P: Anti-Hu-associated paraneoplastic limbic encephalitis in Hodgkin lymphoma. Leuk Lymphoma 2012;53:1433-1434. 